\title{
Key Developmental Regulators Suggest Multiple Origins of Pancreatic Beta Cell Regeneration
}

\author{
Hao Wang, ${ }^{*}$ Xiangyong Wei, ${ }^{*}$ Wenchao Shi, Jianbo He, and Lingfei Luo
}

\begin{abstract}
Extensive efforts have been done to try to restore the lost $\beta$ cell mass for the cure of diabetes. Animal models have been established to provide evidences of cellular origins and contextual regulators of $\beta$ cell regeneration. Here, we used a zebrafish $\beta$ cell ablation and regeneration model to investigate $\beta$ cell neogenesis in the first few days after a near-total $\beta$ cell loss. Regeneration of $\beta$ cells first occurred within $7 \mathrm{~h}$ post-treatment. Developmental regulators such as neurod, pdxl, mnxl, and $n k x 2.2 a$ were active in the regenerating $\beta$ cells, while at the same time suggesting different subpopulations of regenerative cellular origins. Using Cre/loxP-based lineage tracing, we showed that intrapancreatic ductal cells resisted to give rise to regenerating $\beta$ cells. Given that transdifferentiation of $\alpha$ cell and $\delta$ cell can regenerate $\beta$ cell, here we have provided further molecular evidence highly suggesting that the regenerating $\beta$ cells originate from multiple cellular origins.
\end{abstract}

Keywords: zebrafish, $\beta$ cell, regeneration, developmental regulator

\section{Introduction}

$\mathbf{T}$ HE ISLETS COMPRISE insulin-expressing $\beta$ cells, glucagonexpressing $\alpha$ cells, somatostatin-expressing $\delta$ cells, and ghrelin-expressing $\varepsilon$ cells. $\beta$ cell loss or failure causes type 1 diabetes. Type 1 diabetic patients can possibly be cured by restoration of $\beta$ cell mass. ${ }^{1}$ Studies on the endocrine pancreas development and $\beta$ cell regeneration have provided optimistic achievements toward efficient restoration of functional $\beta$ cells.

The vertebrate endocrine pancreas develops from dorsal and ventral pancreatic buds. ${ }^{2}$ In zebrafish, a complete primary islet requires both the dorsal and ventral bud-derived endocrine cells. Dorsal bud-derived primary endocrine islet is present as a cluster of islet cells at the level of the fourth somite by $24 \mathrm{~h}$ postfertilization (hpf). Fusion of dorsal and ventral buds completes at around $52 \mathrm{hpf}$, facilitating contribution of ventral bud-derived endocrine cells to the primary islet. $^{3,4}$ The secondary islet in zebrafish is coming from a subpopulation of intrapancreatic ductal (IPD) cells, which are Notch responsive cells (NRCs). ${ }^{5}$ Inhibition of Notch signaling triggers precocious specification of endocrine cells from NRCs, forming secondary islets within the intrapancreatic duct. ${ }^{5-7}$

Further molecular mechanism underlying differentiation of the secondary islets is poorly understood. The primary and secondary endocrine cells have different progenitor origins, so they may not necessarily share the equivalent developing mechanisms.

Three modes of regeneration have been defined according to different cellular origins of regeneration. ${ }^{89}$ First mode, in response to injury, undifferentiated progenitor cells as a self-renewing source proliferate and differentiate to repair the lost cell population. Second mode, the remnant highly specified cells dedifferentiate and then proliferate. Thereafter, the descendants can perform as same as the first mode to repair the lost cell type. Third mode, a remnant cell population can transdifferentiate into the lost cell type. Proliferation, differentiation, dedifferentiation, and transdifferentiation are the key processes for rebuilding the lost structures during regeneration. Regenerative biology is focusing on these processes to provide insights of cell replacement therapies. ${ }^{10}$

Extensive studies have been focused on $\beta$ cell regeneration. $\beta$ cell-specific ablation and regeneration models have been generated in mouse and zebrafish. Using these models, several endogenous origins have been identified to contribute to the restoration of the lost $\beta$ cell mass.

Under the circumstance of partial ablation, surviving $\beta$ cell proliferation contributes to $\beta$ cell regeneration. ${ }^{11}$ In embryonic development and response to pancreatic duct

Key Laboratory of Freshwater Fish Reproduction and Development, Ministry of Education, Laboratory of Molecular Developmental Biology, School of Life Sciences, Southwest University, Beibei, Chongqing, China.

*These authors contributed equally to this work. 
FIG. 1. $\beta$ cell regeneration after a near-total ablation. (A, B) Representative 3D projections of $\beta$ cells in the primary islet of $T g$ (ins: $C F P-N T R)$ larvae from 0 to $48 \mathrm{hpt}$. The larvae treated with DMSO in the control group showed steady expression of CFP in $\beta$ cells of the primary islet $(\mathbf{A}$, $n=30 / 30$; scale bar, $10 \mu \mathrm{m}$ ). The larvae treated with Mtz showed $0 \beta$ cell at 0 hpt. Nascent $\beta$ cells emerged as regeneration proceeded (B, $n=32 / 37$; scale bar, $10 \mu \mathrm{m}$ ). (C) Quantification of nascent $\beta$ cells at different time points post-treatment. The mean values of $\beta$ cell number are indicated $(n=25$ for each time point. Data are expressed as mean \pm s.e.m.). (D-G) Time series of merged and single-channel confocal planes of primary islets treated with DMSO or Mtz. Tg(ins:CFP-NTR) was used to label $\beta$ cells blue with CFP and perform ablation. Tg(neurod:EGFP) marked neurod + cells with GFP. All $\beta$ cells were neurod-positive in both the DMSO-treated control group and the Mtz-treated group. A neurod+ins- cell at $0 \mathrm{hpt}$ showed positive CFP signal in the following $48 \mathrm{~h}$ (white arrows) indicating its commitment to $\beta$ cell fate (D) $n=23 / 23 ; \mathbf{E}, n=17 / 19$; scale bar, $10 \mu \mathrm{m})$. 3D, three-dimensional; CFP, cyan fluorescent protein; DMSO, dimethyl sulfoxide; GFP, green fluorescent protein; Mtz, metronidazole; NTR, nitroreductase; s.e.m., standard error of the mean. Color images are available online.
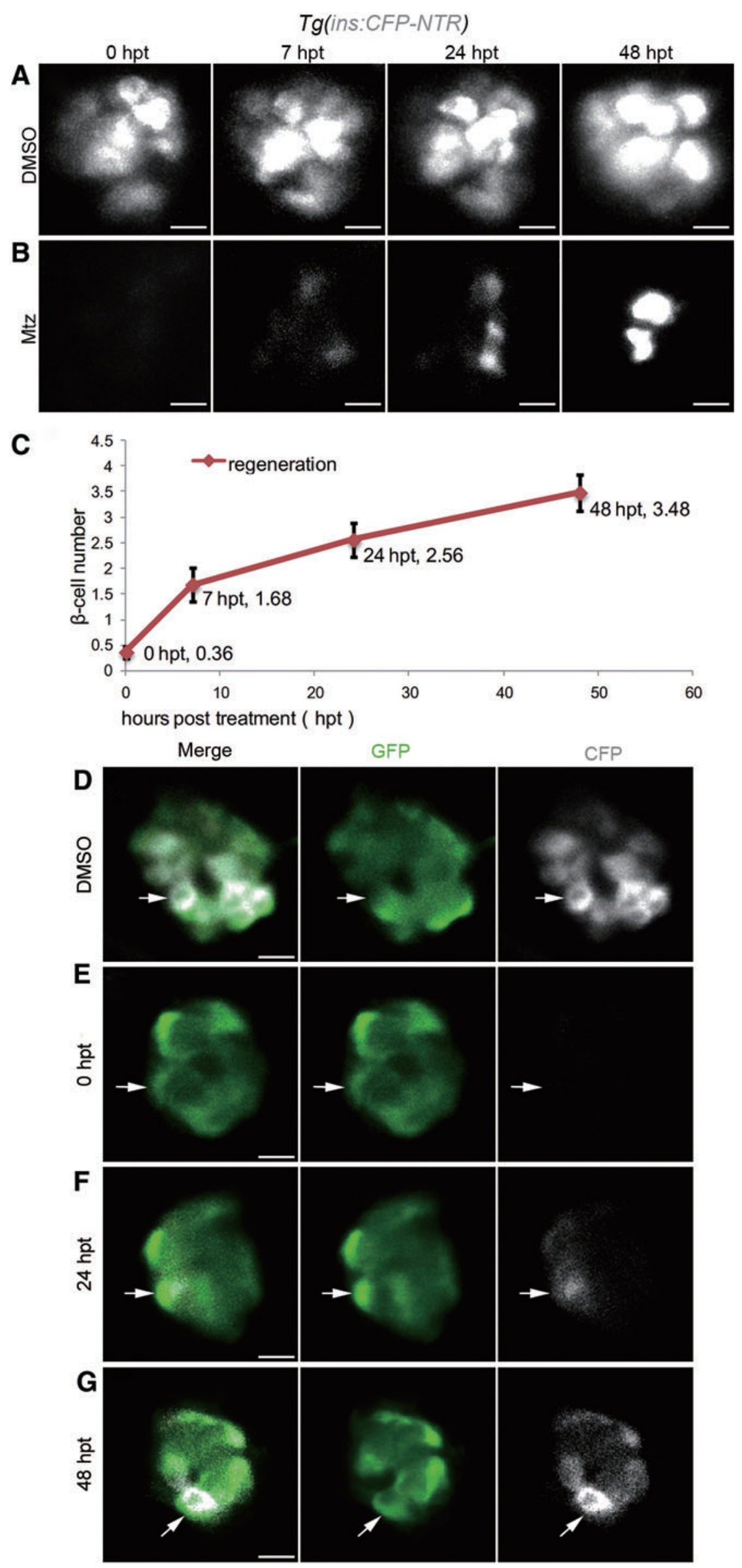

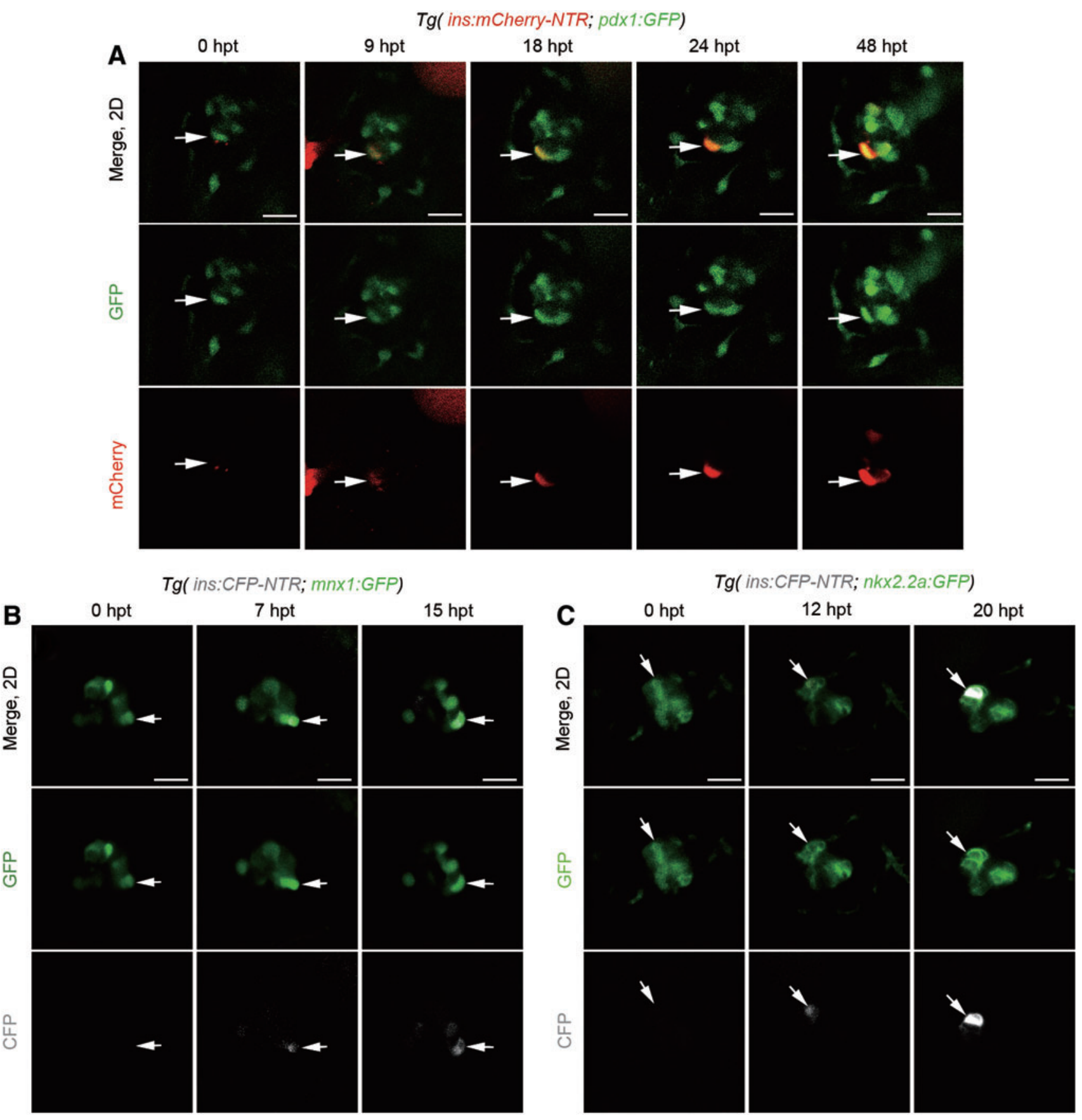

FIG. 2. Nascent $\beta$ cells emerged from pre-existing $p d x 1+, m n x 1+$, or $n k x 2.2 a+$ cells. (A) A set of real-time imaging of a regenerating $\beta$ cell (white arrows) in the primary islet after Mtz treatment using the Tg(ins:mCherry-NTR; pdxl:GFP) transgenic line $(n=19 / 23)$. Merged and single-channel confocal planes showed a pdxl+ins- cell $(0$ hpt, white arrow) committed to the $\beta$ cell fate in the next $48 \mathrm{~h}$. $\beta$ cell maturation was noted by the onset of mCherry fluorescence indicating insulin expression since $9 \mathrm{hpt}$. mCherry expression was getting steadily stronger from 18 to $48 \mathrm{hpt}$, indicating the maintenance of $\beta$ cell identity. (B, C) Time course of a regenerating $\beta$ cell (white arrows) in the primary islet after Mtz treatment using the Tg(mnx1:GFP; ins:CFP-NTR) or Tg(nkx2.2a:GFP; ins:CFP-NTR), respectively. A GFP+CFP-cell (white arrow) showed commitment to the $\beta$ cell fate within several hours after removal of Mtz $(\mathbf{B}, n=21 / 25 ; \mathbf{C}, n=18 / 26$; scale bar, $20 \mu \mathrm{m})$. Color images are available online.

ligation, pancreatic duct-associated progenitors can give rise to nascent $\beta$ cells. ${ }^{12,13}$ Transdifferentiation is a third origin of $\beta$ cell regeneration. Transdifferentiation from $\beta$ cell to $\beta$ cell under extreme $\beta$ cell loss has been reported in both mouse and zebrafish. $^{14,15} \delta$ cell can also convert to $\beta$ cell identity in an age-dependent manner. ${ }^{16}$ Endocrine cells are believed to maintain plasticity to some extent because of their common ancient progenitors while committing to different lineages.

Although several origins of $\beta$ cell neogenesis have been identified, little is known about the mechanisms underlying 
the dedifferentiation/transdifferentiation/differentiation during $\beta$ cell regeneration. Transgenic zebrafish line for targeted $\beta$ cell ablation has been proven to be a rapid and steady ablation/regeneration system, which is a useful tool to address the mechanisms of $\beta$ cell regeneration. ${ }^{17}$ Using this tool, we demonstrate that different cellular origins are implied to simultaneously contribute to the nascent $\beta$ cells to restore the cell mass after a near-total $\beta$ cell loss. $p d x 1, m n x 1$, and $n k x 2.2 a$ are molecular markers that cover all the variant cellular origins. Furthermore, despite the highly suggested multiple origins of $\beta$ cell neogenesis, all the regenerating $\beta$ cells are implied to originate from the neurod-positive endocrine population.

\section{Materials and Methods}

\section{Fish lines}

The zebrafish transgenic lines $T g(p d x l: G F P),{ }^{18} T g B A C$ (neurod:EGFP) $)^{\text {nll }}$ (ref. $^{19}$ ) abbreviated as Tg (neurod:EGFP) ${ }^{\text {nll }}$, $\operatorname{Tg}(m n x 1: G F P)^{m l 2}{\left.\text {, } \text { ref. }^{20}\right)}$ Tg $(-3.5 n k x 2.2 a: G F P)^{i a 3}$ (ref. $\left.^{21}\right)$ abbreviated as $\operatorname{Tg}(n k x 2.2 a: G F P)^{i a 3}$, and Tg(Tplglob:creER $\left.{ }^{T 2}\right)^{j h 12}$ (ref. ${ }^{6}$ ) abbreviated as $T g\left(T p 1: c r e E R^{T 2}\right)^{h 12}$ were previously described. The zebrafish transgenic lines $\operatorname{Tg}(\text { ins:CFP-NTR })^{c q 29}$, $T g$ (ins: $m$ Cherry-NTR $)^{c q 30}$, and Tg(ins:loxP-CFPNTR-stoploxP-DsRed $)^{c q 31}$ were generated in our laboratory. All transgenic lines were raised in $28.5^{\circ} \mathrm{C}$ condition. ${ }^{22-24}$

\section{Treatment of embryos with chemicals}

Larvae were exposed to the following chemicals in egg water. To avoid pigmentation, $0.003 \%$ 1-phenyl-2-thiourea (PTU; Sigma) treatment was applied since 24 hpf. For $\beta$ cell near-full ablation, larvae at $80 \mathrm{hpf}$ were treated with $10 \mathrm{mM}$ metronidazole (Mtz; Sigma) and 0.2\% dimethyl sulfoxide (DMSO) (Sigma) as a carrier for $24 \mathrm{~h}$. After treatment, larvae were washed 3 times with fresh egg water and then recovered in egg water. The control group larvae were treated with equivalent amounts of DMSO under the same conditions. ${ }^{25,26}$

Titrations were performed and the $T g$ (ins:mCherry-NTR) and Tg(ins:loxP-CFPNTR-stop-loxP-DsRed) lines showed similar sensitivity to Mtz treatment as the $T g($ ins:CFP-NTR) line.
For Cre/loxP-mediated lineage tracing during $\beta$ cell regeneration, the larvae of Tg(Tpl:creER ${ }^{T 2}$; ins:loxPCFPNTR-stop-loxP-DsRed) at $80 \mathrm{hpf}$ were treated with $5 \mu \mathrm{M}$ 4-hydroxy-tamoxifen (4-OHT; Sigma), $10 \mathrm{mM} \mathrm{Mtz} \mathrm{(Sigma),}$ and $0.2 \%$ DMSO (Sigma) for $24 \mathrm{~h}$, followed by 3 washes with fresh egg water and recovery in egg water. The control larvae were treated with equivalent amounts of 4-OHT and DMSO under the same conditions. ${ }^{27}$

\section{Fluorescent microscopy and $\beta$ cell counting}

Live embryos were mounted in $1.2 \%$ low melting point agarose (Invitrogen). All images were taken by the Zeiss LSM780 confocal microscope system equipped with ZEN2010 (Carl Zeiss) as previously described. ${ }^{24}$ A $20 \times$ water immersion objective was used. Three-dimensional projections were compiled using ZEN2010. Manual counting of fluorescencepositive cells was performed to determine $\beta$ cell numbers when necessary.

\section{TUNEL assay}

The method of TUNEL assay coupled with whole-mount antibody staining has been described. ${ }^{26}$ The TUNEL assay kit was purchased from Roche (In Situ Cell Death Detection Kit TMR red; Roche).

\section{Fluorescence in situ hybridization and whole-mount antibody staining}

The embryos were fixed overnight in $4 \%$ paraformaldehyde (PFA), the method of fluorescence in situ hybridization (FISH) coupled with whole-mount antibody staining was carried out as previously described ${ }^{26}$ using $m n x 1, p d x 1$ probes, and anti-green fluorescent protein (GFP) antibody (Abcam, ab6658).

\section{Statistical analysis}

Data are expressed as mean \pm standard error of the mean. Statistical significance was tested by two-tailed Student's $t$-test (as noted in the Figure 3M legends).

FIG. 3. Variant key development regulators highly suggest different cellular origins of $\beta$ cell regeneration. (A-L) Merged and single-channel confocal planes of primary islets after DMSO or Mtz treatment. Tg(ins:CFP-NTR) was used to label $\beta$ cell blue with CFP and perform ablation. Single or double transgenic lines were combined to mark specific $\beta$ cell development regulators green with GFP. White arrows indicated GFP-positive $\beta$ cells. White arrowheads indicated GFPnegative $\beta$ cells. Scale bar, $10 \mu \mathrm{m}$. $T g(p d x 1: G F P)$ marked $p d x 1+$ cells. $\beta$ cells in the DMSO-treated control group were all $p d x 1+(\mathbf{A}, n=35 / 35)$. In contrast, some $\beta$ cells were not $p d x 1+$ in the Mtz-treated group $(\mathbf{B}, n=33 / 38)$. Tg $(m n x 1: G F P)$ marked $m n x l+$ cells. Not all $\beta$ cells were $m n x l+$ in either the control or Mtz-treated groups $(\mathbf{C}, n=22 / 22 ; \mathbf{D}, n=22 / 26)$. $T g(n k x 2.2 a: G F P)$ marked $n k x 2.2 a+$ cells. Not all $\beta$ cells were $n k x 2.2 a+$ in either control or Mtz-treated groups $(\mathbf{E}, n=21 /$ $21 ; \mathbf{F}, n=20 / 23)$. $T g(p d x 1: G F P$; $m n x 1: G F P)$ marked $p d x 1+, m n x 1+$, or $p d x 1+m n x 1+$ cells. All $\beta$ cells were GFP positive in both the control and Mtz-treated groups (G, $n=34 / 34 ; \mathbf{H}, n=37 / 37)$. Tg(pdxl:GFP; $n k x 2.2 a: G F P)$ marked $p d x 1+$, $n k x 2.2 a+$, or $p d x 1+n k x 2.2 a+$ cells. All $\beta$ cells were GFP positive in both the control and Mtz-treated groups (II, $n=20 / 20 ; \mathbf{J}$, $n=23 / 25)$. Tg(mnxl:GFP, $n k x 2.2 a: G F P)$ marked $m n x 1+, n k x 2.2 a+$, or $m n x 1+n k x 2.2 a+$ cells. Not all $\beta$ cells were GFP positive in either the control or Mtz-treated groups $(\mathbf{K}, n=21 / 21 ; \mathbf{L}, n=26 / 29)$. (M) Quantifications of ratios of $\beta$ cells that were GFP positive in the primary islets after DMSO or Mtz treatment with listed transgenic lines (DMSO-treated control groups: $m n x 1+, n=5$; all the other groups, $n=3$. Mtz-treated groups: neurod,$+ n=4 ; p d x 1+, n=17 ; m n x 1+, n=8 ; n k x 2.2+$, $n=6 ; p d x 1+, m n x 1+$, or $p d x 1+m n x 1+, n=18 ; p d x 1+, n k x 2.2 a+$, or $p d x 1+n k x 2.2 a+, n=5 ; m n x 1+, n k x 2.2 a+$, or $m n x 1+n k x 2.2 a+, n=8$. Mean \pm s.e.m. All tested by two-tailed Student's $t$-test.). Color images are available online. 

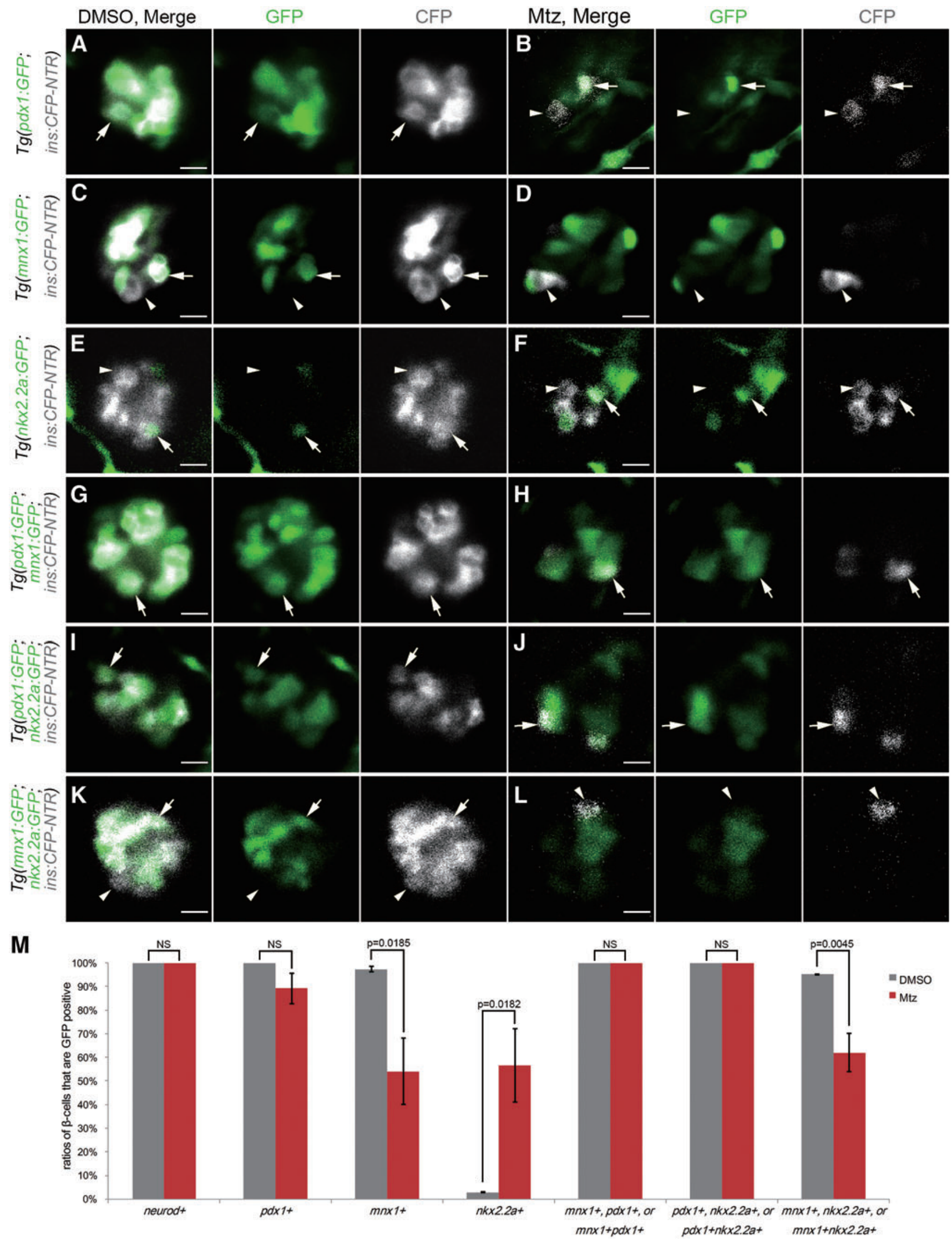


\section{Results}

Nascent $\beta$ cells emerged from the neurod-positive pancreatic endocrine cells

Conditional targeted ablation of cells in zebrafish can be achieved using the nitroreductase (NTR)/Mtz system. ${ }^{26}$ To carry out targeted $\beta$ cell ablation, we generated the Tg(ins:CFP-NTR) ${ }^{c q 29}$ transgenic line, ${ }^{28}$ in which a cassette encoding the cyan fluorescent protein (CFP)-NTR fusion protein was driven by the insulin promoter. Zebrafish larvae were incubated with Mtz from 80 to $104 \mathrm{hpf}$, nearly all the $\beta$ cells were ablated, and $\beta$ cell death was detected during Mtz incubation (Supplementary Fig. S1). One or two nascent $\beta$ cells became detectable $7 \mathrm{~h}$ after the withdrawal of $\mathrm{Mtz}$ (hpt, hours post-treatment) in the majority of larvae (Fig. 1A, B). The number of nascent $\beta$ cells kept increasing at 24 and $48 \mathrm{hpt}$ (Fig. 1C).

To further understand the cellular origins and molecules involved in $\beta$ cell regeneration, different transgenic backgrounds were applied. Instead of the critical roles of $n g n 3$ in the differentiation of multiple pancreatic endocrine cell types in mouse, neurod, together with ascllb, acts as key regulators to control endocrine cell development in zebrafish. ${ }^{29}$ In the Tg(neurod:EGFP; ins:CFP-NTR) double transgenic line, ${ }^{19}$ $100 \%$ of the nascent $\beta$ cells were EGFP-positive cells (Figs. 1D-G and 3M), implying that all the nascent $\beta$ cells are of the neurod-positive endocrine origin.

\section{Combinations of $\mathrm{pdx} 1, \mathrm{mnx} 1$, and $\mathrm{nkx} 2.2 \mathrm{a}$ suggest subpopulations of nascent $\beta$ cells}

To determine whether nascent $\beta$ cells can be divided into subpopulations according to their different cellular origins, other transgenic backgrounds labeling subpopulations of pancreatic cells with GFP were applied. The regenerating $\beta$ cell was characterized by its emergence from the GFPpositive or GFP-negative cells.

Knockdown of $p d x l$ has been reported to cause the reduction of $\beta$ cells at $24 \mathrm{hpf}^{30}$ To explore the contribution of pdxl-positive cells to $\beta$ cell neogenesis, we applied the $T g(p d x 1: G F P)$ transgenic background using another $\beta$ cell ablation line $T g$ (ins: $m$ Cherry-NTR ${ }^{c q 30}$, which exhibited the same performance as the $T g($ ins:CFP-NTR) model. Realtime imaging results showed that at $0 \mathrm{hpt}$, insulin-positive cell was undetectable (Fig. 2A). From 9 to $48 \mathrm{hpt}$, the mCherry-positive cells emerged within the GFP-positive cell population and gradually increased in number, implying contribution of nascent $\beta$ cells from $p d x 1$-positive cells (Fig. 2A). Similar results were also observed under $T g(m n x 1: G F P)^{m l 2}$ and $T g(n k x 2.2 a: G F P)^{i a 3}$ backgrounds (Fig. 2B, C). Statistics showed that $89.2 \%$ of nascent $\beta$ cells were $p d x$ 1:GFP-positive cells (Fig. 3A, B, and M).

Knockdown of $m n x l$ results in partial loss of $\beta$ cells. ${ }^{30}$ $n k x 2.2 a$ regulates $\beta$ cell and $\beta$ cell development in both zebrafish and mouse. ${ }^{21,31}$ Under the $T g(m n x l: G F P)^{m l 2}$ and $\operatorname{Tg}(n k x 2.2 a: G F P)^{i a 3}$ backgrounds, $54.1 \%$ and $56.7 \%$ of regenerating $\beta$ cells were shown to emerge from the mnxlpositive and $n k x 2.2 a$-positive cells, respectively (Fig. $3 \mathrm{C}-\mathrm{F}$ and $\mathrm{M})$. However, in the Tg(mnxl:GFP; $n k x 2.2 a: G F P$; ins: $C F P-N T R)$ transgenic line, only $61.9 \%$ of the regenerating $\beta$ cells were positive for GFP (Fig. $3 \mathrm{~K}$, L, and M), indicating that the majority of $m n x 1$-positive and $n k x 2.2 a$ - positive cells are double positive for $m n x l$ and $n k x 2.2 a$. Since the percentages of nascent $\beta$ cells marked by $p d x 1, m n x 1$, and $n k x 2.2 a$ added up to over $100 \%$, there must be regenerating $\beta$ cells coexpressing two or three of these markers. In the $T g(p d x 1: G F P$; mnxl:GFP; ins:CFP-NTR) or Tg(pdxl:GFP; $n k x 2.2 a: G F P$; ins:CFP-NTR) transgenic line, $100 \%$ of the nascent $\beta$ cells were GFP positive (Fig. 3G-J and M). FISH coupled with antibody staining showed the same pattern with transgenic lines regarding $m n x l$ or $p d x 1$ expression during $\beta$ cell regeneration (Supplementary Fig. S2).

These data suggest that the regenerating $\beta$ cells can be divided into subpopulations according to their expressions of $p d x 1, m n x 1$, and $n k x 2.2 a$. Differential expressions of these developmental regulators suggest variant routes to $\beta$ cell regeneration. Previous studies have reported $\alpha$ cell and $\delta$ cell transdifferentiation as sources of $\beta$ cell regeneration after near-total $\beta$ cell loss. ${ }^{14-16}$ These findings together with our data suggest that $\beta$ cell regeneration is fulfilled through multiple origins. These different subpopulations are suggested to contribute to $\beta$ cell restoration within the same regeneration time window and can be characterized by the expression of different markers.

\section{Pancreatic ductal cells fail to give rise to nascent $\beta$ cells in the primary islet}

Previous studies show that in adult zebrafish $\beta$ cell regeneration, pancreatic ductal cells have an essential contribution to $\beta$ cell regeneration. ${ }^{32,33}$ Because both $p d x 1$ and $n k x 2.2 a$ promoters are active in the endocrine progenitor cells as well as pancreatic ductal cells, contributions of pancreatic ductal cells to regenerating $\beta$ cells were analyzed. The $T g\left(T p 1: c r e E R^{T 2}\right)^{j h 12}$ line expressing 4-OHT-dependent Cre recombinase in the pancreatic ductal epithelium was applied for the Cre/loxP-mediated lineage tracing. ${ }^{5-7,26}$ The Tg(Tp1:CreER ${ }^{T 2}$; ins:loxP-CFPNTR-stop-loxP-DsRed) double transgenic line was generated to simultaneously carry out $\beta$ cell ablation and inducible genetic lineage tracing of nascent $\beta$ cells.

After treatment of the transgenic larvae with 4-OHT and Mtz at the same time from 80 to $104 \mathrm{hpf}$ (Fig. 4A), Notch signaling was activated during targeted $\beta$ cell ablation, for some of the dying $\beta$ cells showed DsRed expression at $0 \mathrm{hpt}$ (Supplementary Fig. S3A). However, none of the nascent $\beta$ cells was positive for DsRed from 15 to $48 \mathrm{hpt}$ (Fig. 4B, C, and Supplementary Fig. S3B-D). These data excluded intrapancreatic ductal cells as cellular origins of regenerating $\beta$ cells in the primary islet during the early stage of development from 4 to $6 \mathrm{dpf}$.

\section{Discussion}

In this study, according to the combinations of different molecular markers, $\beta$ cell regeneration has been highly suggested to originate from different cell types except pancreatic ductal cells. Inside the pancreatic area, nascent regenerated $\beta$ cells are fully marked by the pan-endocrine marker neurod, suggesting limited origins for $\beta$ cell regeneration. Within these neurod-positive cells, transdifferentiation of other endocrine cells and differentiation of quiescent progenitors become the candidate origins of nascent $\beta$ cells.

$p d x 1$ is expressed in the pancreatic progenitors and in differentiated $\beta$ and $\delta$ cells in mouse. ${ }^{34}$ In addition, 


\begin{tabular}{|c|c|c|c|}
\hline $\mathbf{A}$ & $80 \mathrm{hpf}$ & $104 \mathrm{hpf}, 0 \mathrm{hpt}$ & $48 \mathrm{hpt}$ \\
\hline
\end{tabular}

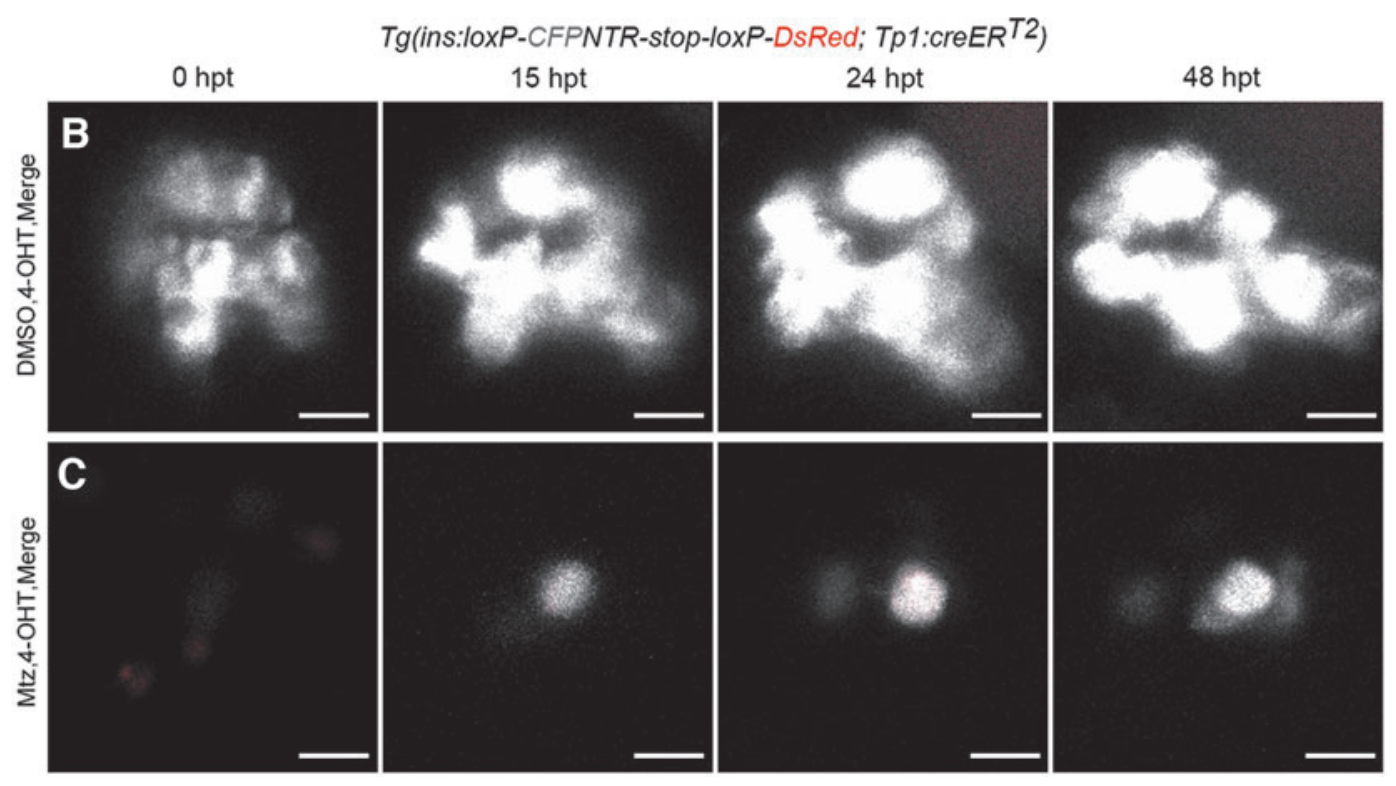

FIG. 4. Pancreatic ductal cells did not contribute to nascent $\beta$ cells. (A) The strategy for Cre/loxP-mediated lineage tracing of pancreatic ductal cells in $\beta$ cell regeneration. 4-OHT was used in both the DMSO-treated control group and Mtztreated group to label Notch-responsive pancreatic ductal cells. Mtz was used only in the Mtz-treated group, but not in the DMSO-treated control group. (B, C) 3D projections of islets in control and Mtz treatment groups from 0 to $48 \mathrm{hpt}$ using Tg(Tp1:creER ${ }^{T 2}$; ins:loxP-CFPNTR-stop-loxP-DsRed) transgenic lines. Notch-responsive pancreatic ductal cells should be labeled by DsRed after 4-OHT treatment. Data showed no $\beta$ cell was positive for DsRed in control group $(\mathbf{B}, n=19 / 19)$. Nascent $\beta$ cells were not positive for DsRed, indicating a negative contribution of $t p 1+$ pancreatic ductal cells to the nascent $\beta$ cells $(\mathbf{C}, n=18 / 23)$. Three $t p 1+$ red fluorescent cells showed in the $(\mathbf{C}) 0 \mathrm{hpt}$ were dying/dead $\beta$ cells with $t p 1$ activated during Mtz treatment. They disappeared thereafter possibly due to cell death. Scale bar, 10 $\mu \mathrm{m}$. 4-OHT, 4-hydroxytamoxifen. Color images are available online.

expression of $p d x 1$ is detectable in pancreatic ductal cells and transdifferentiating $\alpha$ cells in zebrafish. ${ }^{15}$ The zebrafish $m n x l$ is expressed as early as 10-somite stage similar to other early endocrine pancreas genes, ${ }^{35}$ and restricted to $\beta$ cells from $48 \mathrm{hpf}$ on. ${ }^{20}$ Early $n k x 2.2 a$ expression is almost identical to neurod in pancreatic precursors in zebrafish. ${ }^{21}$ In mouse, Nkx2.2 is also present in the early pancreas progenitors, then restricted to $\beta$ cells, a subset of $\alpha$ cells, and PP cells. ${ }^{36}$ In the $T g(n k x 2.2 a: G F P)$ zebrafish line, GFP expression is weak in the primary islet and nearly absent in the $\beta$ cells. $^{21}$

Spontaneous transdifferentiations from $\alpha$ cell $/ \delta$ cell to $\beta$ cell have been reported. ${ }^{14-16}$ In zebrafish, $\delta$ cell transdifferentiation has been excluded as a source of $\delta$ cell regeneration by sst2-cre lineage tracing experiment. ${ }^{15}$ According to the expression pattern of each gene, we deduce that the $p d x 1+n k x 2.2 a+$ or $p d x 1+$ cells might fit the $\alpha$ cell transdifferentiation model. However, because mnxl is not expressed in the $\alpha$ cells, a considerable number of nascent $\beta$ cells originating from $p d x 1+m n x 1+n k x 2.2 a+$ triple-positive cells do not fit any existing model.

One possible origin of these triple-positive cells is the existence of quiescent progenitors. A recent study showed that in adult murine, Dclk1 marked quiescent pancreatic progenitors involved in pancreatic regeneration. ${ }^{37}$ Further Cre/loxP-based lineage tracing under neurod, pdx 1, mnx 1 , or $n k x 2.2 a$ promoter shall substantiate the suggested multiple origins of $\beta$ cell regeneration.

Our results imply that essential regulators of $\beta$ cell development also participate in $\beta$ cell regeneration. How different regulators combine to facilitate $\beta$ cell regeneration still remains an interesting question. Particularly, mechanisms underlying transdifferentiation/differentiation of different cellular origins to $\beta$ cells are of great interest.

\section{Acknowledgment}

We thank Bo Zhang for fish lines.

\section{Disclosure Statement}

No competing financial interests exist.

\section{Funding Information}

This work was supported by the National Key R\&D Program of China (2018YFA0800500), the National Natural Science Foundation of China (31730060, 31970784, 31801214), and the 111 Program (B14037). 


\section{Supplementary Material}

Supplementary Figure S1

Supplementary Figure S2

Supplementary Figure S3

\section{References}

1. Shapiro AM, Lakey JR, Ryan EA, Korbutt GS, Toth E, Warnock GL, et al. Islet transplantation in seven patients with type 1 diabetes mellitus using a glucocorticoid-free immunosuppressive regimen. N Engl J Med 2000;343:230 238.

2. Kinkel MD, Prince VE. On the diabetic menu: zebrafish as a model for pancreas development and function. Bioessays 2009;31:139-152.

3. Field HA, Dong PD, Beis D, Stainier DY. Formation of the digestive system in zebrafish. II. Pancreas morphogenesis. Dev Biol 2003;261:197-208.

4. Lu H, Ma J, Yang Y, Shi W, Luo L. EpCAM is an endoderm-specific Wnt derepressor that licenses hepatic development. Dev Cell 2013;24:543-553.

5. Parsons MJ, Pisharath H, Yusuff S, Moore JC, Siekmann $\mathrm{AF}$, Lawson $\mathrm{N}$, et al. Notch-responsive cells initiate the secondary transition in larval zebrafish pancreas. Mech Dev 2009; 126:898-912.

6. Ninov N, Borius M, Stainier DY. Different levels of Notch signaling regulate quiescence, renewal and differentiation in pancreatic endocrine progenitors. Development 2012; 139:1557-1567.

7. Wang Y, Rovira M, Yusuff S, Parsons MJ. Genetic inducible fate mapping in larval zebrafish reveals origins of adult insulin-producing beta-cells. Development 2011;138: 609-617.

8. Poss KD. Advances in understanding tissue regenerative capacity and mechanisms in animals. Nat Rev Genet 2010; 11:710-722.

9. Tanaka EM, Reddien PW. The cellular basis for animal regeneration. Dev Cell 2011;21:172-185.

10. Shi W, Fang Z, Li L, Luo L. Using zebrafish as the model organism to understand organ regeneration. Sci China Life Sci 2015;58:343-351.

11. Dor Y, Brown J, Martinez OI, Melton DA. Adult pancreatic beta-cells are formed by self-duplication rather than stemcell differentiation. Nature 2004;429:41-46.

12. Inada A, Nienaber C, Katsuta H, Fujitani Y, Levine J, Morita R, et al. Carbonic anhydrase II-positive pancreatic cells are progenitors for both endocrine and exocrine pancreas after birth. Proc Natl Acad Sci U S A 2008;105: 19915-19919.

13. Xu X, D'Hoker J, Stange G, Bonne S, De Leu N, Xiao X, et al. Beta cells can be generated from endogenous progenitors in injured adult mouse pancreas. Cell 2008;132: 197-207.

14. Thorel F, Nepote V, Avril I, Kohno K, Desgraz R, Chera S, et al. Conversion of adult pancreatic alpha-cells to betacells after extreme beta-cell loss. Nature 2010;464:11491154.

15. Ye L, Robertson MA, Hesselson D, Stainier DY, Anderson RM. Glucagon is essential for alpha cell transdifferentiation and beta cell neogenesis. Development 2015;142:14071417.

16. Chera S, Baronnier D, Ghila L, Cigliola V, Jensen JN, Gu $\mathrm{G}$, et al. Diabetes recovery by age-dependent conversion of pancreatic delta-cells into insulin producers. Nature 2014; 514:503-507.

17. Pisharath H, Parsons MJ. Nitroreductase-mediated cell ablation in transgenic zebrafish embryos. Methods Mol Biol 2009;546:133-143.

18. Tehrani Z, Lin S. Antagonistic interactions of hedgehog, Bmp and retinoic acid signals control zebrafish endocrine pancreas development. Development 2011;138: 631-640.

19. Obholzer N, Wolfson S, Trapani JG, Mo W, Nechiporuk A, Busch-Nentwich E, et al. Vesicular glutamate transporter 3 is required for synaptic transmission in zebrafish hair cells. J Neurosci 2008;28:2110-2118.

20. Arkhipova V, Wendik B, Devos N, Ek O, Peers B, Meyer D. Characterization and regulation of the hb9/mnx1 betacell progenitor specific enhancer in zebrafish. Dev Biol 2012;365:290-302.

21. Pauls S, Zecchin E, Tiso N, Bortolussi M, Argenton F. Function and regulation of zebrafish $\mathrm{nkx} 2.2 \mathrm{a}$ during development of pancreatic islet and ducts. Dev Biol 2007; 304:875-890.

22. Liu X, Huang S, Ma J, Li C, Zhang Y, Luo L. NF-kappaB and Snailla coordinate the cell cycle with gastrulation. J Cell Biol 2009;184:805-815.

23. Zou Q, Gang K, Yang Q, Liu X, Tang X, Lu H, et al. The $\mathrm{CCCH}$-type zinc finger transcription factor $\mathrm{Zc} 3 \mathrm{~h} 8$ represses NF-kappaB-mediated inflammation in digestive organs in zebrafish. J Biol Chem 2018;293:11971-11983.

24. Liu C, Wu C, Yang Q, Gao J, Li L, Yang D, et al. Macrophages mediate the repair of brain vascular rupture through direct physical adhesion and mechanical traction. Immunity 2016;44:1162-1176.

25. Cao Z, Mao X, Luo L. Germline stem cells drive ovary regeneration in zebrafish. Cell Rep 2019;26:17091717.e3.

26. He J, Lu H, Zou Q, Luo L. Regeneration of liver after extreme hepatocyte loss occurs mainly via biliary transdifferentiation in zebrafish. Gastroenterology 2014;146: 789-800.

27. Chen J, He J, Ni R, Yang Q, Zhang Y, Luo L. Cerebrovascular injuries induce lymphatic invasion into brain parenchyma to guide vascular regeneration in zebrafish. Dev Cell 2019;49:697-710.e5.

28. Curado S, Anderson RM, Jungblut B, Mumm J, Schroeter E, Stainier DY. Conditional targeted cell ablation in zebrafish: a new tool for regeneration studies. Dev Dyn 2007; 236:1025-1035.

29. Flasse LC, Pirson JL, Stern DG, Von Berg V, Manfroid I, Peers B, et al. Ascl1b and Neurod1, instead of Neurog3, control pancreatic endocrine cell fate in zebrafish. BMC Biol 2013;11:78.

30. Kimmel RA, Onder L, Wilfinger A, Ellertsdottir E, Meyer D. Requirement for Pdx1 in specification of latent endocrine progenitors in zebrafish. BMC Biol 2011; 9:75.

31. Wang J, Elghazi L, Parker SE, Kizilocak H, Asano M, Sussel L, et al. The concerted activities of Pax4 and Nkx2.2 are essential to initiate pancreatic beta-cell differentiation. Dev Biol 2004;266:178-189.

32. Ghaye AP, Bergemann D, Tarifeno-Saldivia E, Flasse LC, Von Berg V, Peers B, et al. Progenitor potential of nkx6.1expressing cells throughout zebrafish life and during beta cell regeneration. BMC Biol 2015;13:70. 
33. Delaspre F, Beer RL, Rovira M, Huang W, Wang G, Gee S, et al. Centroacinar cells are progenitors that contribute to endocrine pancreas regeneration. Diabetes 2015;64:34993509.

34. Offield MF, Jetton TL, Labosky PA, Ray M, Stein RW, Magnuson MA, et al. PDX-1 is required for pancreatic outgrowth and differentiation of the rostral duodenum. Development 1996;122:983-995.

35. Wendik B, Maier E, Meyer D. Zebrafish mnx genes in endocrine and exocrine pancreas formation. Dev Biol 2004; 268:372-383.

36. Sussel L, Kalamaras J, Hartigan-O'Connor DJ, Meneses JJ, Pedersen RA, Rubenstein JLR, et al. Mice lacking the homeodomain transcription factor $\mathrm{Nkx} 2.2$ have diabetes due to arrested differentiation of pancreatic beta cells. Development 1998;125:2213-2221.

37. Westphalen CB, Takemoto Y, Tanaka T, Macchini M, Jiang Z, Renz BW, et al. Dclk1 defines quiescent pancreatic progenitors that promote injury-induced regeneration and tumorigenesis. Cell Stem Cell 2016;18: $441-455$.
Address correspondence to:

Lingfei Luo, PhD

School of Life Sciences

Southwest University

2 Tiansheng Road

Beibei 400715

Chongqing

China

E-mail:1luo@swu.edu.cn

Hao Wang, PhD

School of Life Sciences

Southwest University

2 Tiansheng Road

Beibei 400715

Chongqing

China

E-mail: wanghaowh16@126.com 